Int. J. Dev. Biol. 53: 1349-1358 (2009)

doi: $10.1387 / \mathrm{ijdb} .072421 \mathrm{jc}$

\title{
Genetic control of morphogenesis - Hox induced organogenesis of the posterior spiracles
}

\author{
JAMES CASTELLI-GAIR HOMBRÍA*, MARÍA LUISA RIVAS and SOL SOTILLOS \\ CABD, CSIC/Universidad Pablo de Olavide, Sevilla, Spain
}

\begin{abstract}
The posterior spiracle has become one of the best systems to study how Hox genes control morphogenesis. Interaction of Abdominal-B (ABD-B) with dorso ventral and intrasegmental positional information leads to the local activation of ABD-B primary targets in the dorsal region of the eighth abdominal segment (A8). Primary targets pattern the spiracle subdividing it into two broad areas: external stigmatophore vs. internal spiracular chamber precursor cells. Primary targets then activate secondary targets and modulate the expression of signalling molecules in the spiracle primordium creating unique spiracle positional values. This genetic cascade activates the "realisator" genes that modulate the cell behaviours causing invagination, elongation and cell rearrangements responsible for spiracle morphogenesis. The spiracle realisators that have been identified to date correspond to cell adhesion proteins, cytoskeleton regulators and cell polarity molecules. Interestingly, these realisators localise to different apico-basal locations in the cell (RhoGEF apical, Crumbs subapical, E-cadherin in the adherens junction, RhoGAP basolateral). Therefore, the Hox anterior-posterior code is converted in the cell into apico-basal information required to implement the posterior spiracle morphogenetic program. We believe this may be a common characteristic for Hox induced organogenesis.
\end{abstract}

KEY WORDS: posterior spiracles, ABD-B, Hox gene, morphogenesis, organogenesis

How undifferentiated cells make an organ in a precise location is one of the key questions in Developmental Biology. While certain organs are repeatedly formed along the body axis, others are localised in particular antero-posterior positions by the control of the transcription factors encoded by the Hox genes (also known as homeotic genes). Hox genes activate stable developmental programs that allow the formation of segment specific structures. These structures may consist of particular bones with their unique shapes or of specific organs. An example of Hox gene regulation in mammals is the skeletal structure with its variety of vertebrae shapes and associated ribs. A similar example in arthropods is the variety of leg appendages present along the anterior-posterior axis of the body. In the past fifteen years our laboratory has analysed the posterior spiracles of Drosophila as a model to understand how the morphogenesis of segment specific organs is controlled. Our studies range from the early primordium specification to its final differentiation, trying to find out how the specific behaviour of groups of cells is integrated to achieve the morphogenetic movements that shape the organ. This review summarizes the work of our laboratory and others in what is one of the most complete analyses of how a single Hox gene controls organogenesis.

\section{Posterior spiracle development overview}

The posterior spiracles form the only external connexion of the tracheal respiratory system of the early Drosophila larva. The posterior spiracles are ectoderm-derived epithelial structures composed of two different parts: an internal tube connecting the tracheal dorsal trunk to the outside, and an external protruding structure where the internal tube is lodged (Fig. 1A, B, D, E). The internal tube, or spiracular chamber, forms a cuticular refractile filter (the filzkörper). The external part, or stigmatophore, allows the larva to project the spiracles outside the semi-liquid medium where it feeds. Surrounding the spiracle opening there are four branched sensory hairs.

The spiracles are specified by the Hox gene Abdominal- $B$

Abbreviations used in this paper: A, abdominal segment; Abd-B, Abdominal-B; st, stage.

\footnotetext{
*Address correspondence to: James Castelli-Gair. CABD, CSIC/Universidad Pablo de Olavide, Carretera de Utrera Km 1, 41013 Sevilla. Fax: 34-95-434-9376. e-mail: jcashom@upo.es
} 
Fig. 1. Development of the spiracles. (A) Wild type cuticle of an early larva. (B) Close up of the dorsal A8 segment showing the trachea ( $t$ ) connecting to the base of the spiracle, the filzkörper (fk) formed in the spiracular chamber, the spiracular hairs surrounding the opening of the spiracle (h) and the protruding stigmatophore (st) forming the external part of the spiracle. (C) Close up of an embryo at st11 showing the posterior abdominal segments before the spiracle morphogenetic movements have started. The embryo is stained with anti-pox neuro (POX-N, grey dots) and anti-cut (Ct, brown). Note that POX-N labels a repetitive pattern of sensory neuron precursors in the A5-A8 segments. In contrast, the spiracular chamber precursors labelled by Ct only appear in A8. Arrows point to the invaginating tra-
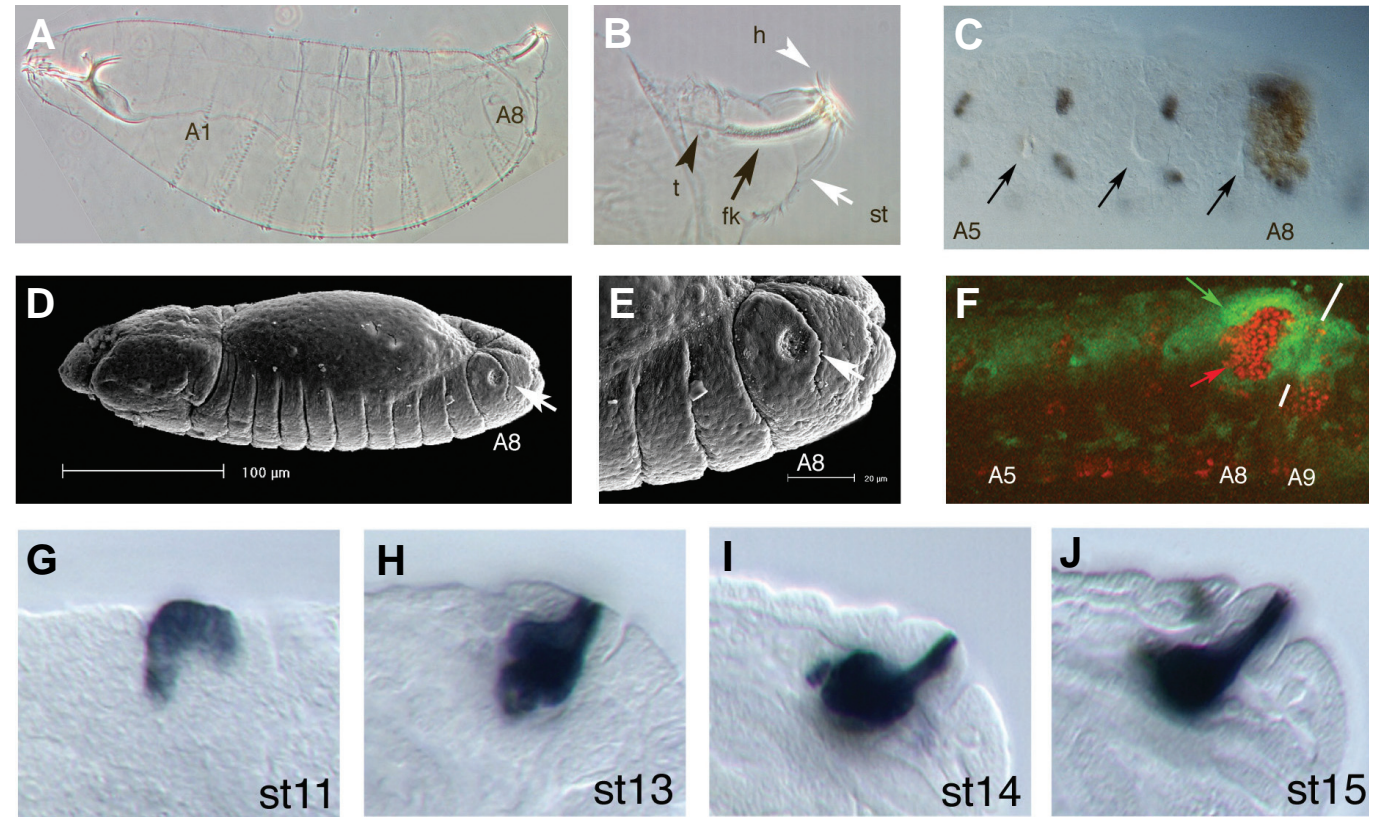

cheal cells, which in A8 are just

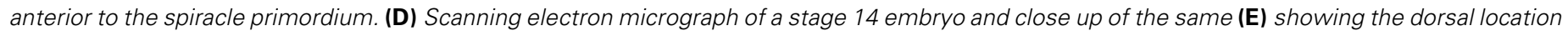

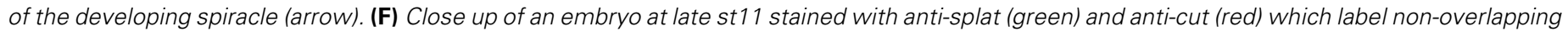

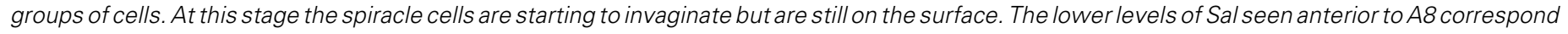

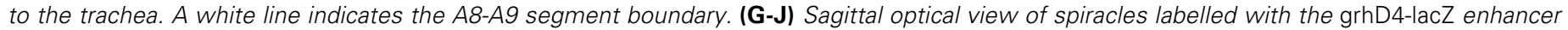

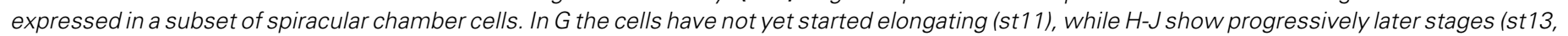
st14, st15). Note in $G$ that some of the labelled cells belong to the tracheal pit. Anterior is left and dorsal up in all panels.

$(A b d-B)$ in the dorsal side of the eighth abdominal segment (A8). At stage 9 before spiracle morphogenesis begins, the primordium of the future posterior spiracle is arranged as a two dimensional sheet of cells located on the dorsal side of A8 just posterior to the cells of the last tracheal pit. Thus, in A8, the tracheal and spiracle primordia are abutting each other (Fig. 1C). At this stage spiracle progenitor cells are morphologically indistinguishable from cells present at homologous positions in segments that do not develop posterior spiracles. ABD-B protein in A8 activates the expression of the first spiracle specific genes. The transcription of these primary spiracle genes is activated in the cells that will form either the internal spiracular chamber or the external stigmatophore. Thus, the spiracle is patterned when the primordium is two dimensional (Fig. 1F) (Hu and Castelli-Gair, 1999).

After this moment (stage 11) the first spiracle specific morphogenetic movements are initiated with the apical cell constriction that leads to the invagination of the future spiracular chamber cells. The spiracle invagination movements proceed from anterior to posterior, starting from the region closer to the tracheal pit. This results in a concerted tracheal pit/ posterior spiracle invagination so that the nascent lumen of the spiracle is always in contact with the A8 tracheal lumen. This contrasts with the formation of the tracheal tree itself, where the invagination of the different tracheal pits arising from each segment is done independently until fusion of these tracheal segments generates a continuous tracheal lumen at stage 14 (Samakovlis et al., 1996). Despite the concerted invagination of the A8 tracheal pit and the posterior spiracle, these two structures use independent developmental programs.

Following the initial invagination step the spiracular chamber goes at stage 13 through a process of cell elongation, where some cells increase the apical basal length up to four fold (Fig. 1 G-J). The spiracle chamber cell elongation is due to an increase of the basolateral membrane relative to the apical membrane (Fig. 2).

The stigmatophore primordium morphogenesis is slightly delayed with respect to that of the spiracular chamber. As the invagination of the spiracular chamber occurs at stage 12 , the surrounding cells of the future stigmatophore rearrange their relative positions by convergent extension movements. This results in the closure of the spiracle opening while simultaneously extending the proximo distal axis of the stigmatophore.

By the end of stage 16 the basic morphogenetic movements of the posterior spiracles have concluded and the three dimensional structure of the spiracles is recognizable (Hu and Castelli-Gair, 1999).

\section{The $A b d-B$ gene}

$A B D-B$ is the only Hox protein required for spiracle specification. $A b d-B$ transcripts are detectable at the blastoderm stage (about 2 hours of development); in contrast, ABD-B proteins are not detectable until the extended germ band stage (about 5 hours of development)(Boulet et al., 1991). This delay in the protein expression may be due to the long transcripts not having time to be completely transcribed during the fast early Drosophila cell cycles leading to the abortive RNAs being degraded as it has been suggested for the UltrabithoraxHox gene (Shermoen and O'Farrell, 1991). $A b d-B$ has a morphogenetic and a regulatory function that can be assigned to two different ABD-B protein isoforms (Fig. 3A): a long $\mathrm{m}$ isoform (for morphogenetic) and a short $r$ isoform (for 
regulatory) (Casanova et al., 1986). Both isoforms contain the DNA binding Homeodomain, but differ in that the ABD-Bm isoform contains an extra 223 amino acid $\mathrm{N}$-terminal region (Celniker et al., 1990, DeLorenzi et al., 1988, Zavortink and Sakonju, 1989).

The isoforms result from the use of different promoters (Fig. $3 \mathrm{~A})$ and are expressed in different spatio-temporal patterns. The $\mathrm{ABD}-\mathrm{Br}$ isoform is expressed in $\mathrm{A} 9$ and posterior segments (Fig. $3 \mathrm{I})$, a region that does not form spiracles, while $A B D-B m$ is expressed in $A 8$, the segment that forms posterior spiracles (Boulet etal., 1991, Delorenzi and Bienz, 1990). Mutants affecting only the ABD-Bm isoform lack spiracles (Fig. 4E and compare $\mathrm{Ct}$ expression in Fig. $3 \mathrm{H}, \mathrm{I})$. Ectopic expression of $\mathrm{ABD}-\mathrm{Bm}$ in segments where ABD-B is not normally present induces supernumerary spiracles (Fig. 3 E,G,J compared to 1D and 3D) (CastelliGair et al., 1994, Kuziora, 1993, Lamka et al., 1992). In contrast, ectopic expression of ABD-Br with the GAL4 system does not form ectopic spiracles (Rivas et al. in preparation) as expected from the absence of spiracles in A9 where ABD-Br is expressed, and the absence of spiracles in Abd-Bm mutants (Fig. 4E) [Surprisingly, heat shock induced ABD-Br activation is also capable of inducing posterior spiracles (Jones and McGinnis, 1993, Kuziora, 1993, Lamka et al., 1992). Our unpublished results indicate that the different effect of expressing Abd-Br with Gal4 or heatshock promoters is due to the heat shock treatment (Rivas et al., in preparation)].

Reflecting their common structure, both isoforms have the capacity to repress transcription of more anterior Hox genes (repression of anterior Hox gene transcription by posterior expressed Hox proteins is a common property of all HOX Drosophila proteins (Struhl and White, 1985)). As with other Hox proteins, it has been shown that the response to ABD-Bm expression varies through development. Ectopic expression of ABD-Bm can only induce the formation of posterior spiracles in the first 5 hours of development (CastelliGair, 1998a). Suggesting that there is a time window after which cells cannot differentiate into posterior spiracles.

The functional difference between both isoforms and the temporal response of cells to ABD-Bm expression suggest a simple model to explain the presence of spiracles exclusively in A8. Although Abd-Bm is expressed in A5-A8 its expression is temporally regulated, with $\mathrm{ABD}-\mathrm{Bm}$ first detected at st9 in A8 (when cells are competent to make spiracles) and later expanding to A5-A7 (Fig. $3 \mathrm{~B}, \mathrm{C})$. Thus the Abd-Bm expression anterior to $A 8$ occurs at a time when the cells are refractory to posterior spiracle induction (Castelli-Gair, the top in all panels. 1998a). The absence of spiracles in $A 9$ is explained by the inability of the $\mathrm{Abd}-\mathrm{Br}$ isoform to activate in wild type conditions the spiracle downstream targets (Rivas et al., in preparation).

Posterior spiracles are only formed on the dorsal side of the A8 segment. This dorsal restriction is likely to be due to interaction of ABD-Bm with the dorsoventral patterning system mediated by the TGF $\beta$ pathway. The Dpp morphogen (a Drosophila TGF $\beta$ homolog) is expressed dorsally in the embryo. Interaction between the TGF $\beta$ pathway effectors with $A B D-B m$ is likely to result in activation of spiracle genes in the dorsal part of A8, as ectopic expression of $d p p$ or an activated TGF Receptor at 5 hours results in the unrestricted activation of spiracle genes in an A8 dorso ventral stripe (Rivas et al., in preparation).

\section{$A b d-B$ transcriptional regulation}

As described above, the formation of the spiracles requires the correct temporal and spatial activation of the Hox $A b d-B$ gene in the posterior abdominal segments. The cis regulatory region of $A b d-B$ is extremely complex with enhancers, silencers, boundary and maintenance regulatory elements spread over 100 kilobases upstream and downstream of the transcribed region (reviewed in Akbari et al., 2006). Analysis of the non-coding sequences have resulted in the finding of several elements named infra abdominal
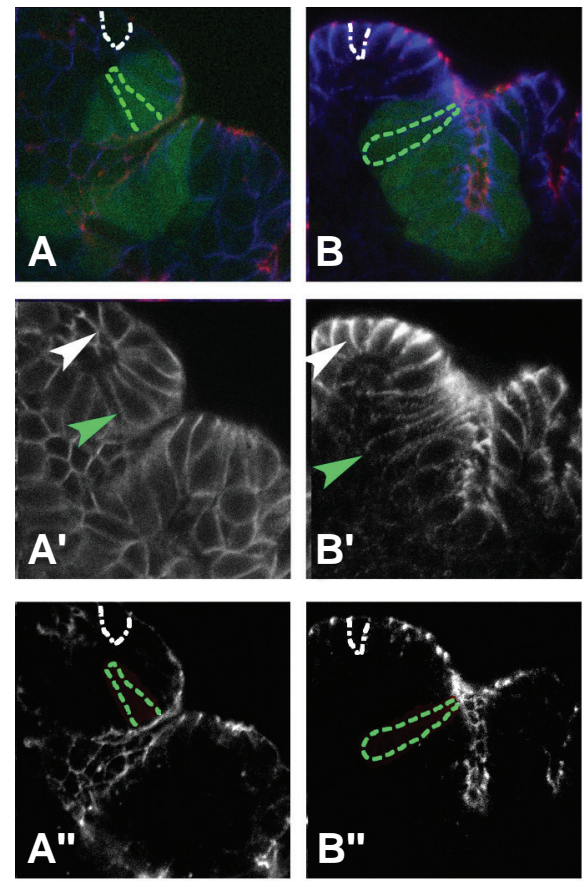

st13
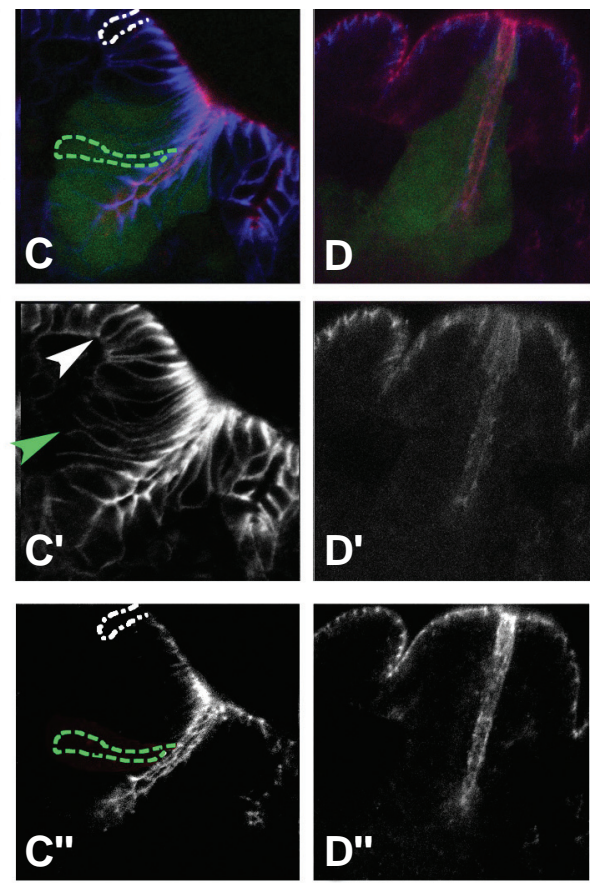

st15

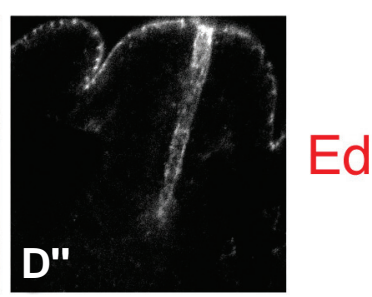

st16

Fig. 2. The relative amount of apical vs. basal cell membrane varies during spiracle cell elongation. Triple labeling of ems-Gal4 UAS-GFP embryos stained with cell polarity markers at progressively later stages: (A) st13, (B) st14, (C) st15 and (D) st16. A subset of spiracle chamber cells is labelled with GFP (green). The apical region of the cells is labelled with anti-Echinoid (red), the basolateral membrane with anti-Scribble (blue). Some cells have been highlighted to drive attention to the relative apical vs. basal membrane surface changes occurring in the spiracular chamber cells (green dotted lines or arrowhead) compared to the neighbouring epidermal cells (white dotted line or arrowhead). The middle row (A'-D') shows separately Scrb staining and the lower row (A"'-D") Echinoid. Anterior is to the left and dorsal to 
(iab), capable of driving expression in the Abd-B domain. iab elements are flanked by Fab boundary elements required to isolate their domains of action (Barges et al., 2000, Mihaly et al., 2006, Zhou et al., 1999, Zhou et al., 1996, Zhou and Levine, 1999). Of particular relevance for spiracle development are a 3 ' $i a b-8$ and a 5' $i a b-8$ element able to drive expression in the A8 segment from stage 9 (Estrada et al., 2002).

Not much is known about the upstream transcriptional regulators of $A b d-B$. Mutations in any of the maternal components required for the antero posterior definition of the embryo result in the formation of embryos possessing two perfect posterior spiracles in the anterior end (Fig. 4B), however these transformations are likely to be indirect with antero-posterior genes not having a direct input on $A b d-B$ transcription.

After the definition of the antero posterior axis, the embryo is subdivided into segments by the gap and pair-rule genes. These genes participate in the spatial activation of the Hox genes. Antibody staining and RNA in situ detection show that the GAP genes knirps and Krüppe/act as $A b d-B$ repressors in the anterior abdomen (Casares and Sanchez-Herrero, 1995), however the cuticles of such embryos do not form ectopic spiracles suggesting that they may contribute to the late pattern of $A b d-B$ transcription. The expression of $A b d-$ $B$ in $A 8$ and $A 9$ requires the function of the terminal gene tailless ( $t / I)$. In $t / /$ mutant embryos the A8 segment is missing as well as the more terminal elements of the telson (Fig. 4C) (Harding and Levine, 1988). Although TII is a direct regulator of the Hox gene Ultrabithorax (Qian et al., 1993) there is no direct evidence proving that TIl directly activates $A b d-B$ transcription. In any

Fig. 3. The Hox gene Abd-B is required for posterior spiracle formation. Schematic representation of the Abd-B genomic region and transcripts giving rise to the different protein isoforms (A). The black line represents genomic DNA with the approximate position of the iab-8 cis regulatory elements represented by red boxes. Light blue boxes represent non-coding exons, dark blue boxes coding regions and a red star the homeobox. Four promoters give rise to four transcripts one of which encodes the $A B D-B m$ protein and the other three an identical shorter $\mathrm{ABD}-\mathrm{Br}$ isoform. (B) Expression of both isoforms at stage 11 stained with an antibody against a common exon. Arrows in A6-A7 point to a patch of ABD-B expressed in these segments. (C) A similar stage embryo double stained with anti $A b d-B$ and anti-en to show that the high levels of $A B D-B$ occur in A8-A9. (D) Close up of a stage 11 embryo stained with the apical marker aPKC showing the connexion between the spiracle and the trachea. (E) Scanning electron micrograph of a st12 embryo expressing ubiquitously Abd-Bm showing the formation of ectopic spiracles in each trunk segment (arrows). (G) St14 embryo expressing ubiquitously Abd-Bm and stained with the apical marker E-cad showing the formation of ectopic spiracles in A4-A6 segments. (J) Cuticle of an embryo of the same genotype as in $(E, G)$ showing the ectopic spiracles (arrow) formed anterior to the normal ones (arrowhead). (F) Close up of the embryo shown in (C) but rotated $180^{\circ}$ to compensate for the inversion caused by the germ band extension. Arrowhead in (F) points at the dorsal cells that form the spiracle. (H) St11 wild type embryo stained with anti-Ct to show the position where the spiracular chamber forms (arrowhead). (I) St11 Abd-B ${ }^{M 5}$ embryo lacking the $A B D-B m$ isoform stained with anti-ABD-B (brown) and anti-Ct (grey). The brown staining shows the expression of $A B D-B r$ which is confined to the posterior compartment of $A 8$ and to $A 9$ (compare with $F$ where both $m$ and $r$ isoforms are expressed). Arrowhead points at the position where Ct positive cells marking the spiracle primordium are located in the wild type (compare with H). case, as TII is expressed both in the anterior and the posterior ends of the embryo, additional genes must modulate the posterior activation of $A b d-B$.

Maintenance of $A b d-B$ expression is negatively regulated by the Polycomb $(\mathrm{Pc})$ group of chromatin regulators. However, the ectopic expression of $A b d-B$ caused in $P C$ or extrasexcomb mutants does not result in well developed ectopic spiracles (Lawrence et al., 1983), probably due to the ectopic ABD-B expression appearing after the 5 hour time interval when cells
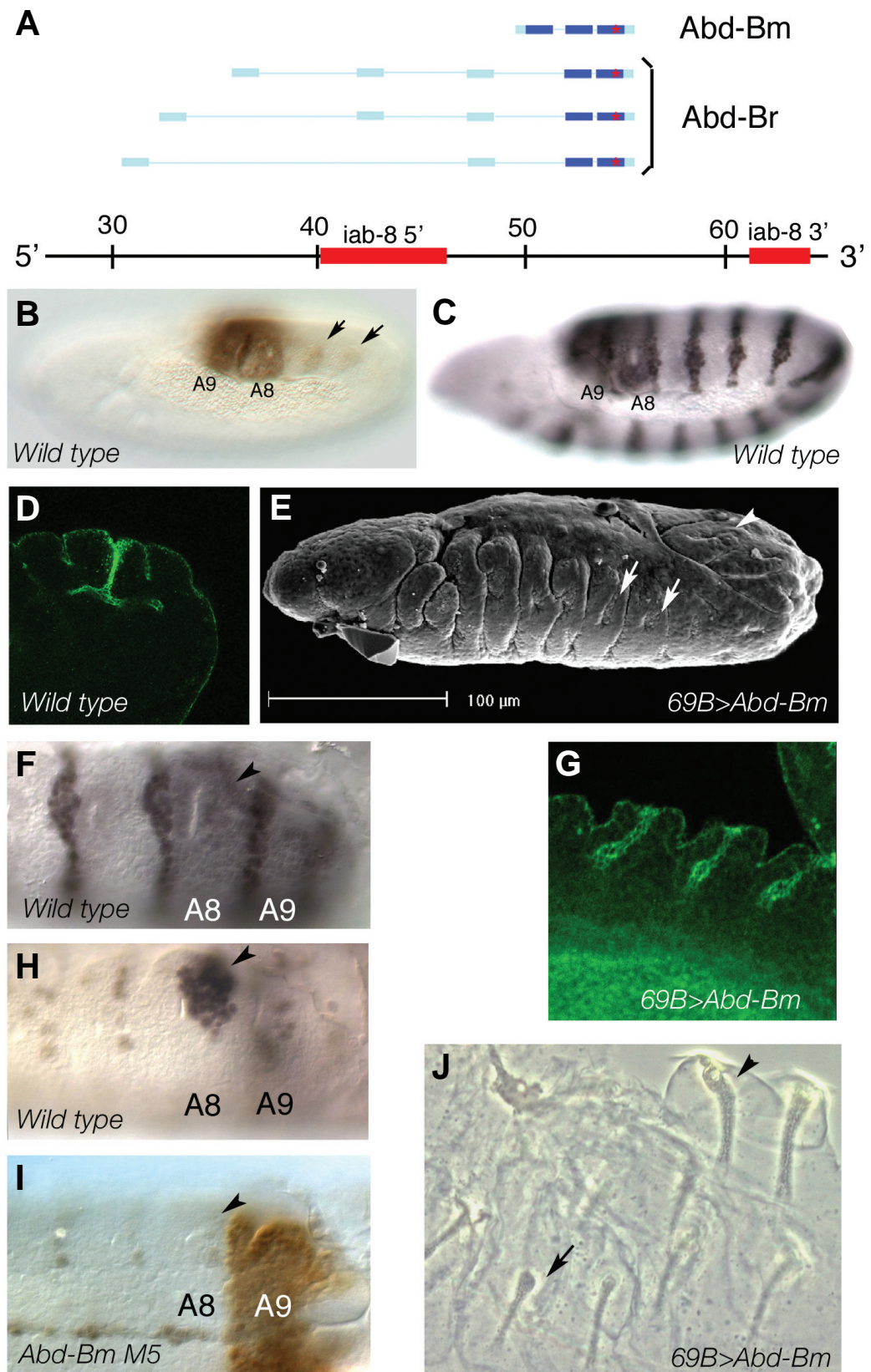
are competent to form posterior spiracles.

\section{Modulation of ABD-B function}

The function of many Hox proteins is modulated by co-factor interaction. Co-factors modify the functional outcome of a protein without affecting its expression. The DNA binding affinity of the HOX proteins Labial, Deformed, Sex combs reduced, Ultrabithorax and Abdominal-A is modulated by their interaction with the extradenticle (EXD) and Homothorax (HTH) homeodomain proteins (Henderson and Andrew, 2000, Mann, 1995, Peifer and Wieschaus, 1990, Rieckhof et al., 1997), but neither EXD nor HTH are thought to play a major role on ABD-B DNA binding or function (van Dijk and Murre, 1994).

The only gene that has been shown to modify the ABD-B functional outcome without affecting its levels of expression is lines (lin) (Castelli-Gair, 1998b). Lines is a protein with conserved domains with no evident functions (Hatini et al., 2000). lin is expressed ubiquitously and is required for $\mathrm{ABD}-\mathrm{Bm}$ to activate all of its known spiracle targets, but not required for the repression of anterior Hox genes [(Castelli-Gair, 1998b) and Rivas etal., unpublished]. As a result, lin mutant embryos form an A8 segment with no cuticular structures due to a lack of Hox input (Fig. 4D). Lin is not required for the function of other Hox genes suggesting its Hox related activity is $A b d$ $B$ specific, and probably ABD-Bm specific (CastelliGair, 1998b).

$\mathrm{Lin}$ is also required in regions where $A B D-B$ is not expressed. Studies of Hox independent Lin functions have shown that Lin binds to the Bowl and Drumstick (Drm) zinc finger proteins. In the gut and in the dorsal epidermis, the three proteins participate in a linear relief of repression pathway in which Lines decreases the levels of Bowl protein except on those cells that also express Drm. Drm N-terminal zinc finger binds Lin and competes for Bowl binding, liberating Bowl from the post translational regulation exerted on it by Lin (Hatini et al., 2005). Although bowl and drm mutant embryos do not present any obvious posterior spiracle defects, future work should address if a similar relationship between Drm, Bowl and Lin is also involved in A8 development.

\section{Spiracle patterning by ABD-B primary targets}

The posterior spiracles are specified at stage 10, when the spiracle primordium is still two-dimensional. The first targets to be expressed in a spiracle specific pattern are the homeodomain encoding genes empty spiracles (ems) and cut(ct); and the ligand of the JAK/STAT pathway unpaired (upd) (Hu and Castelli-Gair, 1999, Lovegrove et al., 2006) (Fig. 5A-C). All these genes are expressed in the future spiracular chamber in partially overlapping patterns and their expression does not depend of each other's expression, suggesting that their enhancers are independently activated by ABD-B and intrasegmental signals. In contrast, the spalt (sa) gene (encoding a zinc finger protein) is expressed in the stigmatophore cells in a complementary pattern to that of Ct (Fig. 1F and 5C, D). This complementary expression is achieved by $\mathrm{Ct}$ repression of sa/transcription and may be one of the principal mechanisms subdividing the spiracle primordium in internal (spiracular chamber) vs. external (stigmatophore) regions. This subdivision is the first indication of posterior spiracle patterning (Hu and Castelli-Gair, 1999). Direct activation of the above genes by ABD-B has only been studied for ems (Jones and McGinnis, 1993, Taylor, 1998), but their early temporal expression and independence from other regulators suggests they are all direct targets. Thus, we will refer collectively to the early spiracle targets as primary ABD-B targets.

The expression of $c t$ in the posterior spiracles is regulated by a complex set of enhancers (Jack and DeLotto, 1995), one is dedicated to the posterior spiracle hair precursors, another one dedicated to three patches of stigmatophore chamber cells, and there must exist at least an unidentified enhancer controlling cts early posterior spiracle expression. A single ems spiracle enhancer has been identified whose expression is activated from the earliest stages. This enhancer binds ABD-B in vitro and is regulated by ABD-B in vivo(Jones and McGinnis, 1993, Taylor, 1998). The upd and upd2 genes encoding the two main ligands of the Drosophila JAK/STAT pathway are also expressed in the posterior spiracles under ABD-B regulation (Hombría et al., 2005, Lovegrove et al., 2006, and S. Brown unpublished results). upd and ems expression in the posterior spiracles stops at st13 (Fig. 
$5 \mathrm{E}, \mathrm{F})$. In contrast to this transient expression, ctand sa/expression in the posterior spiracles is maintained throughout embryogenesis (Fig. 5C, D, G, H).

Mutation of the ABD-B primary targets, cause different defects in the spiracles (Hu and Castelli-Gair, 1999). sa/mutants form a spiracular chamber but lack the stigmatophore, in agreement with Sal's restricted expression to the stigmatophore. On the contrary, $c t$ and ems mutations affect exclusively the development of the spiracular chamber. In ems mutants the cells closer to the tracheal pit do not invaginate staying on the embryo's surface. This causes the trachea to disconnect from the posterior spiracle and results in the absence of filzkörper and spiracle lumen. In ct mutants the trachea remains attached to the spiracle and a lumen forms although the spiracles lack the filzkörper almost completely. In ct mutants the spiracular hairs are missing, showing that besides its function on spiracle morphogenesis, $\mathrm{Ct}$ is required to specify the spiracle's peripheral nervous derivatives. Mutations blocking the signal transduction elements of the DrosophilaJAK/ STAT pathway (either mutation of the three Upd ligands; the receptor dome; the JAK kinase hop; or the gene encoding the Drosophila STAT transcription factor stat92E) cause a lack of spiracle cell elongation (Lovegrove et al., 2006). As a result the spiracular chamber cells remain on the embryo's surface displacing the stigmatophore. The stigmatophore displacement may be indirectly caused by the absence of spiracular chamber morphogenesis, but it cannot be discarded that STAT is directly required for the stigmatophore morphogenesis as the Upd ligands could diffuse from the spiracular chamber primordium activating STAT in the stigmatophore cells.

Simultaneous mutation of the four primary targets results in the complete absence of posterior spiracles. Conversely, ectopic activation of Ct, Upd, Ems and the Sal downstream target Grn (see below) induces expression of posterior spiracle downstream targets in ectopic positions (Lovegrove et al., 2006). These defects are similar to what happens after loss of function of $A b d$ $B$ or ectopic $A b d-B$ induction respectively (Castelli-Gair et al., 1994, Lamka etal., 1992, Sánchez-Herrero etal., 1985), suggesting that these four genes are the only ABD-B primary targets required during posterior spiracle induction (Lovegrove et al., 2006).

Besides these four primary targets other putative primary targets, like Klumpfuss and nubbin (nub/pdm-1), are expressed in the early spiracle primordium. However, as their mutation does not affect spiracle development, it is unclear if they are required for spiracle development at all, or if their expression in the spiracle primordium is an evolutionary remnant ( $\mathrm{Hu}$ and Castelli-Gair, 1999).

Given that the primary targets encode transcription factors (or induce local activation of transcription factor as is the case of STAT activation by the Upd ligands) it follows that their main function is to activate a tier of secondary targets.

\section{ABD-B secondary targets}

Secondary targets are activated from stage 11 onwards. Secondary targets include other transcription factors and signalling molecules, implying there will be also higher order targets as morphogenesis unfolds. The exact number of higher order targets is unknown but it may be large, as the BDGP systematic RNA expression database described 140 genes whose expression is modulated in the posterior spiracles (Lovegrove PhD thesis). Given the difficulty to classify these higher order targets as secondary, tertiary or higher, and the possibility of feed back regulatory loops that would make this classification absurd, we will refer to them collectively as secondary targets. We favour this name rather than "indirect $A B D-B$ targets" as we cannot exclude that in some cases their expression would simultaneously require $\mathrm{ABD}-\mathrm{B}$ and one of ABD-B's primary targets.

Secondary targets may be regulated by a single ABD-B primary target, or by a combination of them. As the primary targets are expressed in partially overlapping patterns this results in secondary targets activated in different subsets of cells, which confers cellular heterogeneity to the spiracle. As many of these secondary targets may be expressed in the spiracles but be irrelevant for the spiracle's development or physiology, we will only consider in this review secondary targets that, when mutant, cause spiracle defects.

The expression of some secondary targets is relatively specific to the posterior spiracles, as is the case of the multi-zinc finger protein DmOAZ (Krattinger et al., 2007). DmOAZtranscription is activated by a combination of Ems and Ct. Other secondary targets are expressed in various tissues but are up-regulated through a spiracle specific enhancer. This is the case for the transcription factor encoded by grainy head (grh/E/f-1) (Fig. $1 \mathrm{G}$ J) (Bray and Kafatos, 1991, Lovegrove et al., 2006), or the spiracle expression of the tracheal determinant trachealess (trh) (Isaac and Andrew, 1996). Grh is expressed in the embryonic epidermis and in the trachea. In the latter, Grh has been shown to limit the amount of apical membrane (Hemphala et al., 2003). Although the specific function of grhin spiracles has still not been determined, the grhspiracle enhancer labels a group of cells with extremely elongated shapes and small apical membrane domains (Fig. 1G-J) suggesting that Grh spiracle up-regulation may be responsible for the small apical membrane domain formed by these cells. trh mutants lack trachea and have short filzkörpers. The spiracle phenotype may be a direct function on the spiracular chamber and not a secondary defect due to the absence of trachea, as the trachea are also defective in $\mathrm{vV} /$ mutants which form a long filzkörper (de Celis et al., 1995).

The main secondary target genes downstream of Sal in the stigmatophore are the transcription factor GATAc encoded by grain (grn) (Fig. 5L) and the homeobox gene engrailed (en). Grn is expressed and required in the stigmatophore and the spiracular chamber. Grn mutants have spiracles with shorter spiracular chambers and with stigmatophores almost absent. This second phenotype is due to the absence of cell rearrangements of the spiracular chamber (Brown and Castelli-Gair Hombría, 2000). Given that Grn also controls cell rearrangements during leg evertion in the femur and tibia of the fly (Brown and Castelli-Gair Hombría, 2000), it is possible that the internal defects are also due to a defect in cell rearrangements in the stigmatophore.

The engene is normally expressed in the posterior compartment of each segment. However, in A8 Sal induces entranscription in dorsal anterior cells (Merabet et al., 2005). This local respecification of the anterior A8 cells may be necessary to translate the antero-posterior positional values of the segment to the circular values of the stigmatophore. en mutant embryos have specific defects on spiracle development that can be separated 
from those caused by En's prior requirement for embryonic segmentation (Merabet et al., 2005).

Some signalling molecules are also secondary targets of the cascade, in a process that serves to generate cell diversity in the posterior spiracle cells (see next section).

The function of ABD-B in this transcription factor cascade could be exclusively involved on the activation of primary targets but could also be involved in the activation of secondary targets. The observation that spiracle-like structures can be formed in the absence of $A b d-B$ by simultaneous ectopic expression of the primary targets, suggests that the role of $A b d-B$ for secondary target activation is minor (Lovegrove et al., 2006). However ABD$B$ function at later stages is required for maintaining the repression of more anterior Hox transcription factors in A8. This repressive function is important to maintain spiracle development (Rivas et al., in preparation).

\section{Modulation of intrasegmental patterning cues by Abd-B}

The molecules controlling the Hedgehog (Hh), WNT and Epidermal Growth Factor (EGF) signalling pathways constitute an important class of ABD-B secondary targets. Most of the components of these pathways were originally classified as segment polarity genes because they are required for the normal segmentation of the embryo. In fact, many of them are expressed during segmentation in striped segmental patterns. However, the segmental expression patterns of wingless (wg), rhomboid (rho) and en which respectively encode a WNT ligand, a protease required for EGF ligand processing, and the transcription factor regulating hh expression, change in $A 8$ after $A B D-B$ activation. These changes occur at st 11 preceding any morphogenetic movements and are not caused by the morphological changes arising in A8 (Merabet et al., 2005). The A8 specific regulation of these signalling molecules is important for spiracle development, as blocking the function of these signalling pathways during the morphogenetic stages causes severe spiracle defects. This A8 segment specific modulation is exemplified by the disappearance of the normal dorsal wgpatch of expression and its substitution by $2 \mathrm{wg}$ expressing cells. Similarly, a second patch of rhoexpression appears in A8 in addition to the normal rho stripe. As previously described, en becomes activated in the anterior compartment of A8 downstream of Sal. Therefore, ABD-B controlled modulation of these molecules remodels the intrasegmental positional information in A8 helping to form the posterior spiracles (Merabet et al., 2005).

\section{Posterior spiracle realisator genes}

Hox realisator genes have been defined as the downstream target genes responsible for controlling the local cell behaviours (cell adhesion, shape, etc) that induce the morphogenesis of particular segment specific organs (García-Bellido, 1975). Several spiracle realisator genes have been identified which can be grouped in three classes: cell adhesion proteins, cytoskeleton regulators and cell polarity proteins. In all cases analysed, the expression of the realisator gene is controlled downstream of the primary targets, suggesting that ABD-B controls indirectly the spiracle realisators (Lovegrove et al., 2006).

\section{Cell adhesion proteins}

Cadherins are a super family of Calcium-dependent cell adhesion proteins. All cadherins are transmembrane proteins containing several cadherin repeats in the extra cellular domain. The intracellular domain varies among the various cadherins. In the classic cadherin family it contains a $\beta$-catenin binding domain that is absent in the non-classic cadherins. The expression of four non-classic cadherins (cad86C, cad74A, cad88C and cad96C) and of the classical Epithelial-cadherin (E-cad) gene is specifically regulated in the posterior spiracles.

$E$-cad is expressed ubiquitously in the ectoderm. However, higher levels of expression are induced in the posterior spiracles by JAK/STAT activation and Ct. In zygotic mutant embryos for $E$ cad, where normal development proceeds until the formation of the ectoderm, the spiracles fail to invaginate in a proportion of
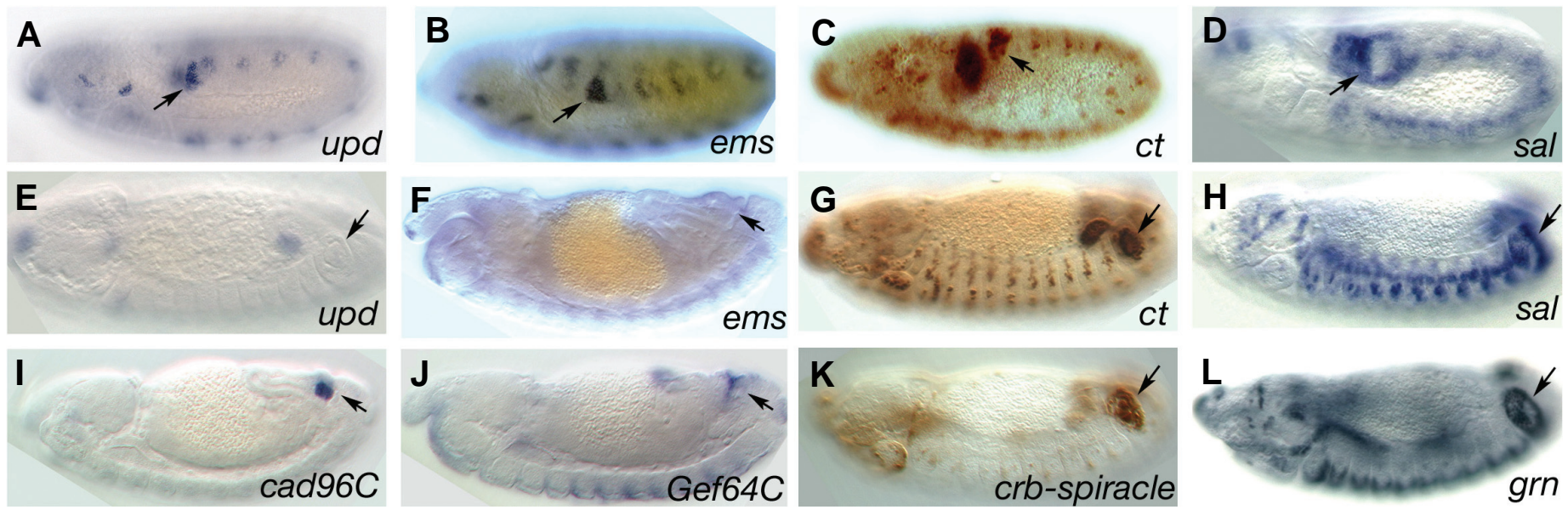

Fig. 5. Expression of ABD-B downstream targets. Primary targets at early stages (A-D) or late stages (E-H). While upd and ems expression ceases after st 13, ct and sal are continuously expressed on the spiracular chamber and the stigmatophore respectively. The secondary target grn (L) has a similar stigmatophore expression pattern to its upstream regulator sal (H). (I-K) Expression of three ABD-B realisators. (I) The non-classical cad-96C is expressed in the internal spiracle cells. (J) Expression of Gef64C RNA. Note that the apical localisation of the RNA highlights the spiracle lumen. (K) Expression of the spiracle enhancer located in the first crb intron. Arrows point to the spiracle. (A-D) are stage 10-11 embryos, (E-L) are stage 13-14. 
embryos. The uninvaginated cells remain on the ectoderm surface where they still manage to go through the second phase of cell elongation and form a filzkörper like cuticle (Lovegrove et al., 2006).

During development, cad86C, cad74A, cad88C and cad96C are mainly expressed in the posterior spiracles (Fig. $5 \mathrm{I}$ and (Lovegrove et al., 2006)). Detailed analysis of the region of expression using FISH double in siturevealed that these cadherins are expressed in a mosaic distribution on different subsets of spiracle cells. cad86C is expressed in the distal cells of the stigmatophore, cad $74 A$ in the distal cells of the spiracular chamber, and cad88C and cad96C are co-expressed in the most internal spiracular chamber cells (Lovegrove et al., 2006). Using a Cad74A-GFP fusion protein it has been confirmed in cell culture that this non-classic cadherin is capable of mediating Ca-dependent cell adhesion (Simoes et al., 2006). Double antibody staining with $\beta$-catenin and Cad74A-GFP showed that Cad74A localises apical to the adherens junction, thus E-cad and Cad74A localise to different membrane compartments on the apical side of the cell. Injection of dsRNA for the non-classic cadherins expressed in the spiracles resulted in a significant RNA decrease, but no spiracle phenotypes were observed. This is also the case for simultaneous injection of dsRNA for two cadherins. However, simultaneous injection of dsRNA for cad88C and cad96C in E-cad zygotic mutants significantly increases the number of embryos with abnormal spiracle invagination compared to the zygotic E-cad control embryos. This result indicates that the non-classic cadherins have a minor role on spiracle development (Simoes et al., 2006).

Another molecule with cell adhesion properties, Neurotactin (Nrt), is initially expressed at homogeneous levels in the ectoderm, but by st14 Nrt expression disappears from most of the ectoderm, remaining at high levels on the posterior spiracles. The Nrt ligand, Amalgam, can also be detected at high levels on spiracle cells (Fremion et al., 2000, Liebl et al., 2003). The function of Nrt in this system is not clear as nrt mutants do not show overtly abnormal spiracles (Lovegrove et al., 2006).

\section{Cytoskeletal regulators}

During cell invagination and cell elongation there is a major reorganisation of the actin and tubulin cytoskeletons. The ubiquitously expressed family of small Rho GTPases (Including Rho, Rac, Cdc42) constitutes the most important cytoskeleton regulators. Rho GTPases switch from an active GTP to an inactive GDP bound form. When active, the GTPases bind target proteins that are responsible for the cytoskeleton modulation. The posterior spiracle cytoskeleton is regulated by Rho GTPases as shown by the lack of spiracle invagination observed in Rac and Rho mutants and the spiracle defects caused by the expression of constitutive activated or dominant negative forms of Rho GTPases (Denholm et al., 2005, Simoes et al., 2006).

Using a probe that exclusively binds the active RhoGTP fraction, it has been observed that during spiracle development active Rho1 accumulates in the cell's apical region (Simoes et al., 2006). A well-known downstream target of active Rho1 is the Myosin II regulatory light chain (MRLC, encoded by the spaghetti squash gene). As expected from the apical location of RhoGTP, MRLC relocalises from basolateral to apical cell compartments during the stages of spiracle invagination. Similarly, Myosin II heavy chain and Actin also localise apically in the spiracles.
These observations and the fact that spiracles do not invaginate properly in MRLC mutants, suggests that a contractile apical actomyosin structure is required for spiracle invagination (Simoes et al., 2006).

The above results indicate that the subcellular organisation of the cytoskeleton is finely regulated in spiracle cells by RhoGTPases. The connexion between the ABD-B regulatory cascade and the ubiquitously expressed GTPases is found at the level of the GTPase regulators. The RhoGAP (Rho GTPase Activating Proteins) and the RhoGEF (Rho Guanine Exchange Factors) proteins control the GTP-GDP cycle. RhoGEFs activate the GTPases by displacing the GDP molecule allowing Rho to bind the excess of GTP present in the cytoplasm. RhoGAPs repress by binding to RhoGTP and enhancing its GTPase activity.

At least two RhoGEFs function during spiracle morphogenesis (Lovegrove et al., 2006, Simoes et al., 2006). RhoGEF64C is specifically activated in the spiracles downstream of ABD-B primary targets, thus linking a GEF to ABD-B (Fig. 5J). RhoGEF64C RNA and protein localise to the apical side of the spiracle cell, the area where active Rho is detected. Although genetic interaction shows that RhoGef64C interacts with Rho1 in the spiracles, mutations of $R$ hoGef64C result in a mild spiracle phenotype suggesting that other Rho activators are present. In fact, analysis of RhoGef2 mutant embryos reveals they have strong spiracle invagination defects (Simoes et al., 2006). In contrast to RhoGef64C, RhoGef2expression is ubiquitous. However, analysis of the distribution of RhoGEF2 in the posterior spiracle reveals that the ABD-B cascade regulates the protein's subcellular localisation, which shifts from the basal to the apical side. The regulation of RhoGEF2 subcellular localisation is not exclusive for the spiracles as it also happens during mesoderm invagination (Kolsch et al., 2007).

Mutation of the RhoGAP crossveinless-c ( $c v-c)$ gene affects posterior spiracle invagination (Denholm et al., 2005). In the ectoderm $c v-c$ is expressed in the tracheal primordia of all segments with higher levels in A8, where its up-regulation depends on ABD-B (Lovegrove et al., 2006). Interestingly, GFP fusion proteins show that $\mathrm{Cv}$-c protein localises to the basolateral membrane domain in a complementary pattern to that of the GEFs (Simoes et al., 2006). This suggests that Rho-GTP apical accumulation is achieved by the complementary effect of GEF mediated apical activation and GAP mediated basolateral repression. All the above results show that the correct subcellular localisation of the GAP and GEF regulators in the spiracle cells must be tightly controlled during morphogenesis.

\section{Cell polarity regulators}

Cell shape changes in polarised epithelial cells modify the relative amounts of apical vs. basal membrane surface. This is particularly evident when using antibodies specific to proteins that localise to specific membrane domains on the elongating spiracle cells (Fig. 2). Staining of spiracle cells with the septate junction protein Disc large (DIg), reveals an expansion in the basolateral membrane of spiracle cells. The expansion of the basolateral membrane is even more evident when staining with the basolateral proteins Nrt or Scribble (Scrb). Analysis of the apical proteins Ecad, Echinoid (Ed) and Crumbs (Crb) reveals that the apical membrane surface constricts during invagination. In the spiracle, apical surface reduction is accompanied by an increase in the 
expression levels of some of these proteins. Especially interesting is the case of Crb. Crb is a conserved transmembrane protein that localises apical to the adherens junction as detected with $\mathrm{E}$ cadherin. Crb is part of a protein complex necessary for maintaining cell polarity. In crbmutant embryos the loss of cell polarity is shown by the loss of correctly localised E-cad and a subsequent epithelial collapse (Grawe et al., 1996). On the other hand, ectopic expression of $\mathrm{Crb}$ protein results in the mislocalisation of Crb to the basolateral membrane, also causing E-cad mislocalisation and epithelial collapse (Klebes and Knust, 2000). Thus, correct Crb subcellular localisation is fundamental for maintaining cell polarity. Analysis of crb expression reveals that besides its ubiquitous ectoderm expression, higher levels of protein and RNA are detected in the spiracle primordium. crbmutants have shorter spiracles and expression of a Crb dominant negative protein results in abnormal spiracles that in a fraction of embryos resemble those formed in JAK/STAT mutants. Analysis of the crb cis regulatory region reveals that in the first intron there is a spiracle specific enhancer directly regulated by STAT (Fig. 5K) (Lovegrove et al., 2006).

These results show that during spiracle cell invagination and elongation, proteins controlling different membrane domains have to be tightly regulated. ABD-B is indirectly regulating the expression of the apical membrane determinant Crb through the activation of upd in the posterior spiracle primordium. This results in STAT activation that leads to increased transcription of $c r b$. It is not yet clear how higher levels of crbtranscription in the spiracles control elongation. Crb may be involved in the maintenance of the apical membrane at a stage when the basolateral membrane is elongating. Alternatively, increased Crb levels may help the subcellular relocalisation of the RhoGEFs cytoskeleton regulators or of other proteins to the apical membrane.

In summary, our thorough understanding of how the posterior spiracles of Drosophila are formed makes them a superb model to analyse how Hox induced genetic cascades control morphogenesis at the cellular level, a system that could be used to complement modern genomic and proteomic systems biology approaches.

\section{Acknowledgements}

J. C. -G. H. is indebted to past members of his laboratory (S. Brown, N. Hu and B. Lovegrove) and collaborators (B. Denholm, H. Skaer, M. Zeidler, Y. Graba, S. Merabet, S. Simões and A. Jacinto). The spiracle project would not have been possible without the initial encouragement of MichaelE. Akam and A. Martínez-Arias, the support of The Royal Society and The Wellcome Trust and more recently of the Ministerio de Educación y Ciencia and Junta de Andalucía.

\section{References}

AKBARI, O.S., BOUSUM, A., BAE, E. and DREWELL, R.A. (2006). Unraveling cisregulatory mechanisms at the abdominal-A and Abdominal-B genes in the Drosophila bithorax complex. Dev Bio/293: 294-304.

BARGES, S., MIHALY, J., GALLONI, M., HAGSTROM, K., MULLER, M., SHANOWER, G., SCHEDL, P., GYURKOVICS, H. and KARCH, F. (2000). The Fab-8 boundary defines the distal limit of the bithorax complex iab-7 domain and insulates iab-7 from initiation elements and a PRE in the adjacent iab-8 domain. Development 127: 779-90.

BOULET, A.M., LLOYD, A. and SAKONJU, S. (1991). Molecular definition of the morphogenetic and regulatory functions and the cis-regulatory elements of the Drosophila Abd-B homeotic gene. Development 111: 393-405.
BRAY, S.J. and KAFATOS, F.C. (1991). Developmental function of Elf-1: an essential transcription factor during embryogenesis in Drosophila. Genes Dev 5: 1672-83.

BROWN, S. and CASTELLI-GAIR HOMBRÍA, J. (2000). Drosophila grain encodes a GATA transcription factor required for cell rearrangement during morphogenesis. Development 127: 4867-76.

CASANOVA, J., SANCHEZ-HERRERO, E. and MORATA, G. (1986). Identification and characterization of a parasegment specific regulatory element of the abdominal-B gene of Drosophila. Cel/ 47: 627-36.

CASARES, F. and SANCHEZ-HERRERO, E. (1995). Regulation of the infraabdominal regions of the bithorax complex of Drosophila by gap genes. Development 121: 1855-66.

CASTELLI-GAIR, J. (1998a). Implications of the spatial and temporal regulation of Hox genes on development and evolution. Int J Dev Bio/42: 437-44.

CASTELLI-GAIR, J. (1998b). The lines gene of Drosophila is required for specific functions of the Abdominal-B HOX protein. Development 125: 1269-1274.

CASTELLI-GAIR, J., GREIG, S., MICKLEM, G. and AKAM, M. (1994). Dissecting the temporal requirements for homeotic gene function. Development 120: 198395.

CELNIKER, S.E., SHARMA, S., KEELAN, D.J. and LEWIS, E.B. (1990). The molecular genetics of the bithorax complex of Drosophila: cis-regulation in the Abdominal-B domain. EMBO J9: 4277-86.

DE CELIS, J.F., LLIMARGAS, M. and CASANOVA, J. (1995). Ventral veinless, the gene encoding the $\mathrm{C} f 1$ a transcription factor, links positional information and cell differentiation during embryonic and imaginal development in Drosophila melanogaster. Development 121: 3405-16.

DELORENZI, M., ALI, N., SAARI, G., HENRY, C., WILCOX, M. and BIENZ, M. (1988). Evidence that the Abdominal-B r element function is conferred by a trans-regulatory homeoprotein. EMBO J7: 3223-31.

DELORENZI, M. and BIENZ, M. (1990). Expression of Abdominal-B homeoproteins in Drosophila embryos. Development 108: 323-9.

DENHOLM, B., BROWN, S., RAY, R.P., RUIZ-GOMEZ, M., SKAER, H. and HOMBRÍA, J.C. (2005). crossveinless-c is a RhoGAP required for actin reorganisation during morphogenesis. Development 132: 2389-400.

ESTRADA, B., CASARES, F., BUSTURIA, A. and SANCHEZ-HERRERO, E. (2002). Genetic and molecular characterization of a novel iab-8 regulatory domain in the Abdominal-B gene of Drosophila melanogaster. Development 129: 5195-204.

FREMION, F., DARBOUX, I., DIANO, M., HIPEAU-JACQUOTTE, R., SEEGER, M.A. and PIOVANT, M. (2000). Amalgam is a ligand for the transmembrane receptor neurotactin and is required for neurotactin-mediated cell adhesion and axon fasciculation in Drosophila. EMBO J19: 4463-72.

GARCÍA-BELLIDO, A. (1975). Genetic control of wing disc development in Drosophila. Elsevier, Amsterdam.

GRAWE, F., WODARZ, A., LEE, B., KNUST, E. and SKAER, H. (1996). The Drosophila genes crumbs and stardust are involved in the biogenesis of adherens junctions. Development 122: 951-9.

HARDING, K. and LEVINE, M. (1988). Gap genes define the limits of antennapedia and bithorax gene expression during early development in Drosophila. EMBO J: 205-14.

HATINI, V., BOKOR, P., GOTO-MANDEVILLE, R. and DINARDO, S. (2000). Tissue- and stage-specific modulation of Wingless signaling by the segment polarity gene lines. Genes Dev 14: 1364-76.

HATINI, V., GREEN, R.B., LENGYEL, J.A., BRAY, S.J. and DINARDO, S. (2005). The Drumstick/Lines/Bowl regulatory pathway links antagonistic Hedgehog and Wingless signaling inputs to epidermal cell differentiation. Genes Dev 19: 70918

HEMPHALA, J., UV, A., CANTERA, R., BRAY, S. and SAMAKOVLIS, C. (2003) Grainy head controls apical membrane growth and tube elongation in response to Branchless/FGF signalling. Development 130: 249-58.

HENDERSON, K.D. and ANDREW, D.J. (2000). Regulation and function of Scr, exd, and hth in the Drosophila salivary gland. Dev Bio/217: 362-74.

HOMBRÍA, J.C., BROWN, S., HADER, S. and ZEIDLER, M.P. (2005) Characterisation of Upd2, a Drosophila JAK/STAT pathway ligand. Dev Biol 288: $420-33$ 
HU, N. and CASTELLI-GAIR, J. (1999). Study of the posterior spiracles of Drosophila as a model to understand the genetic and cellular mechanisms controlling morphogenesis. Developmental Biology 214: 197-210.

ISAAC, D.D. and ANDREW, D.J. (1996). Tubulogenesis in Drosophila: a requirement for the trachealess gene product. Genes Dev 10: 103-17.

JACK, J. and DELOTTO, Y. (1995). Structure and regulation of a complex locus: the cut gene of Drosophila. Genetics 139: 1689-700.

JONES, B. and MCGINNIS, W. (1993). The regulation of empty spiracles by Abdominal-B mediates an abdominal segment identity function. Genes Dev7: 229-40.

KLEBES, A. and KNUST, E. (2000). A conserved motif in Crumbs is required for Ecadherin localisation and zonula adherens formation in Drosophila. Curr Bio/10: 76-85.

KOLSCH, V., SEHER, T., FERNANDEZ-BALLESTER, G.J., SERRANO, L. and LEPTIN, M. (2007). Control of Drosophila gastrulation by apical localization of adherens junctions and RhoGEF2. Science 315: 384-6.

KRATtINGER, A., GENDRE, N., RAMAEKERS, A., GRILLENZONI, N. and STOCKER, R.F. (2007). DmOAZ, the unique Drosophila melanogaster OAZ homologue is involved in posterior spiracle development. Dev Genes Evo/217: 197-208.

KUZIORA, M.A. (1993). Abdominal-B protein isoforms exhibit distinct cuticular transformations and regulatory activities when ectopically expressed in Drosophila embryos. Mech Dev 42: 125-37.

LAMKA, M.L., BOULET, A.M. and SAKONJU, S. (1992). Ectopic expression of UBX and $A B D-B$ proteins during Drosophila embryogenesis: competition, not a functional hierarchy, explains phenotypic suppression. Development 116: 84154.

LAWRENCE, P.A., JOHNSTON, P. and STRUHL, G. (1983). Different requirements for homeotic genes in the soma and germ line of Drosophila. Cel/ 35: 2734.

LIEBL, E.C., ROWE, R.G., FORSTHOEFEL, D.J., STAMMLER, A.L., SCHMIDT, E.R., TURSKI, M. and SEEGER, M.A. (2003). Interactions between the secreted protein Amalgam, its transmembrane receptor Neurotactin and the Abelson tyrosine kinase affect axon pathfinding. Development 130: 3217-26.

LOVEGROVE, B., SIMOES, S., RIVAS, M.L., SOTILLOS, S., JOHNSON, K., KNUST, E., JACINTO, A. and HOMBRÍA, J.C. (2006). Coordinated control of cell adhesion, polarity, and cytoskeleton underlies Hox-induced organogenesis in Drosophila. Curr Bio/16: 2206-16.

MANN, R.S. (1995). The specificity of homeotic gene function. BioEssays 17: 85563.

MERABET, S., HOMBRÍA, J.C., HU, N., PRADEL, J. and GRABA, Y. (2005). Hoxcontrolled reorganisation of intrasegmental patterning cues underlies Drosophila posterior spiracle organogenesis. Development 132: 3093-102.
MIHALY, J., BARGES, S., SIPOS, L., MAEDA, R., CLEARD, F., HOGGA, I., BENDER, W., GYURKOVICS, H. and KARCH, F. (2006). Dissecting the regulatory landscape of the Abd-B gene of the bithorax complex. Development 133: 2983-93.

PEIFER, M. and WIESCHAUS, E. (1990). Mutations in the Drosophila gene extradenticle affect the way specific homeo domain proteins regulate segmental identity. Genes Dev 4: 1209-23.

QIAN, S., CAPOVILLA, M. and PIRROTTA, V. (1993). Molecular mechanisms of pattern formation by the BRE enhancer of the Ubx gene. EMBO J12: 3865-77.

RIECKHOF, G.E., CASARES, F., RYOO, H.D., ABU-SHAAR, M. and MANN, R.S. (1997). Nuclear translocation of extradenticle requires homothorax, which encodes an extradenticle-related homeodomain protein. Cel/91: 171-83.

SAMAKOVLIS, C., HACOHEN, N., MANNING, G., SUTHERLAND, D.C., GUILLEMIN, K. and KRASNOW, M.A. (1996). Development of the Drosophila tracheal system occurs by a series of morphologically distinct but genetically coupled branching events. Development 122: 1395-407.

SHERMOEN, A.W. and O'FARRELL, P.H. (1991). Progression of the cell cycle through mitosis leads to abortion of nascent transcripts. Ce//67: 303-10.

SIMOES, S., DENHOLM, B., AZEVEDO, D., SOTILLOS, S., MARTIN, P., SKAER, H., HOMBRÍA, J.C. and JACINTO, A. (2006). Compartmentalisation of Rho regulators directs cell invagination during tissue morphogenesis. Development 133: 4257-67.

STRUHL, G. and WHITE, R.A.H. (1985). Regulation of the Ultrabithorax gene of Drosophila by the other bithorax complex genes. Cel/43: 507-519.

SÁNCHEZ-HERRERO, E., VERNÓS, I., MARCO, R. and MORATA, G. (1985). Genetic organisation of the Drosophilabithorax complex. Nature313: 108-113.

TAYLOR, H.S. (1998). A regulatory element of the empty spiracles homeobox gene is composed of three distinct conserved regions that bind regulatory proteins. Molecular Reproduction and Development 49: 246-253.

VAN DIJK, M.A. and MURRE, C. (1994). extradenticle raises the DNA binding specificity of homeotic selector gene products. Cel/78: 617-24.

ZAVORTINK, M. and SAKONJU, S. (1989). The morphogenetic and regulatory functions of the Drosophila Abdominal-B gene are encoded in overlapping RNAs transcribed from separate promoters. Genes Dev 3: 1969-81.

ZHOU, J., ASHE, H., BURKS, C. and LEVINE, M. (1999). Characterization of the transvection mediating region of the abdominal-B locus in Drosophila. Development 126: 3057-65.

ZHOU, J., BAROLO, S., SZYMANSKI, P. and LEVINE, M. (1996). The Fab-7 element of the bithorax complex attenuates enhancer-promoter interactions in the Drosophila embryo. Genes Dev 10: 3195-201.

ZHOU, J. and LEVINE, M. (1999). A novel cis-regulatory element, the PTS, mediates an anti-insulator activity in the Drosophila embryo. Ce//99: 567-75. 


\section{Further Related Reading, published previously in the Int. J. Dev. Biol.}

See our recent Special Issue Fertilization, in honor of David L. Garbers and edited by Paul M. Wassarman and Victor D. Vacquier at: http://www.ijdb.ehu.es/web/contents.php?vol=52\&issue=5-6

PBX proteins: much more than Hox cofactors

Audrey Laurent, Réjane Bihan, Francis Omilli, Stéphane Deschamps and Isabelle Pellerin

Int. J. Dev. Biol. (2008) 52: 9-20

Patterning and morphogenesis of the vertebrate inner ear

Jinwoong Bok, Weise Chang and Doris K. Wu

Int. J. Dev. Biol. (2007) 51: 521-533

$\mathrm{XSu}(\mathrm{H}) 2$ is an essential factor for gene expression and morphogenesis of the Xenopus gastrula embryo

Motoaki Ito, Tomohisa Katada, Seiji Miyatani and Tsutomu Kinoshita

Int. J. Dev. Biol. (2007) 51: 27-36

Hox and ParaHox genes in Nemertodermatida, a basal bilaterian clade

Eva Jiménez-Guri, Jordi Paps, Jordi García-Fernández and Emili Saló

Int. J. Dev. Biol. (2006) 50: 675-679

Pulling forces acting on Hox gene clusters cause expression collinearity

Spyros Papageorgiou

Int. J. Dev. Biol. (2006) 50: 301-308

PBX1 intracellular localization is independent of MEIS1 in epithelial cells of the developing female genital tract

Agnès Dintilhac, Réjane Bihan, Daniel Guerrier, Stéphane Deschamps, Héloise

Bougerie, Tanguy Watrin, Georgette Bonnec and Isabelle Pellerin

Int. J. Dev. Biol. (2005) 49: 851-858

Additional enhancer copies, with intact $c d x$ binding sites, anteriorize Hoxa-7/lacZ expression in mouse embryos: evidence in keeping with an instructional cdx gradient

Stephen J. Gaunt, Adam Cockley and Deborah Drage

Int. J. Dev. Biol. (2004) 48: 613-622

Evolution of the Hox/ParaHox gene clusters.

David E K Ferrier and Carolina Minguillón

Int. J. Dev. Biol. (2003) 47: 605-611

Conserved genetic mechanisms for embryonic brain patterning.

Heinrich Reichert

Int. J. Dev. Biol. (2002) 46: 81-87

In vitro induction systems for analyses of amphibian organogenesis and body patterning.

T Ariizumi and M Asashima

Int. J. Dev. Biol. (2001) 45: 273-279

Study of the downstream network of Abd-B involved in spiracle development in Drosophila.

J Castelli-Gair

Int. J. Dev. Biol. (1996) 40: S75-S76

Modeling the regulation of the bithorax complex in Drosophila melanogaster: the phenotypic effects of Ubx, abd-A and Abd-B heterozygotic larvae, and a homozygous Ubx- abd A hybrid gene.

M Carratalá, I Vernós, R Ransom and R Marco

Int. J. Dev. Biol. (1989) 33: 455-466

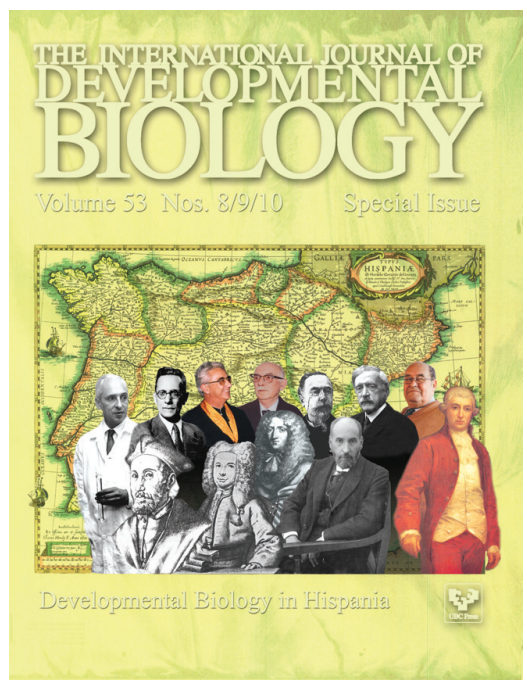

5 yr ISI Impact Factor $(2008)=3.271$

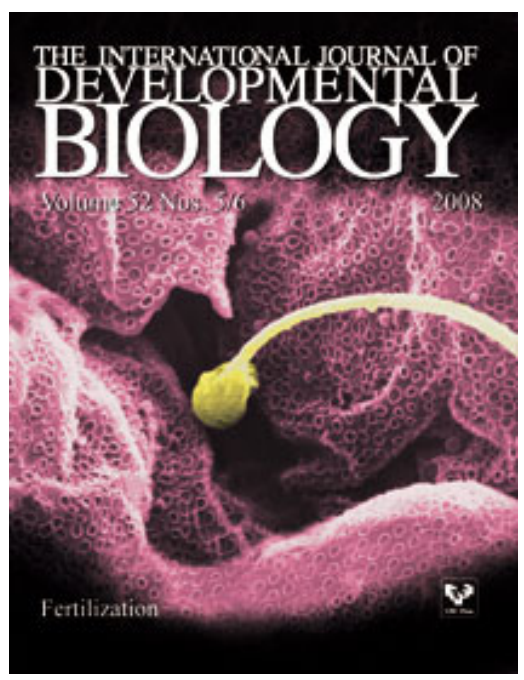

